\title{
Situs Inversus: A Laparoscopic Observation
}

Key words: situs inversus, laparoscopy

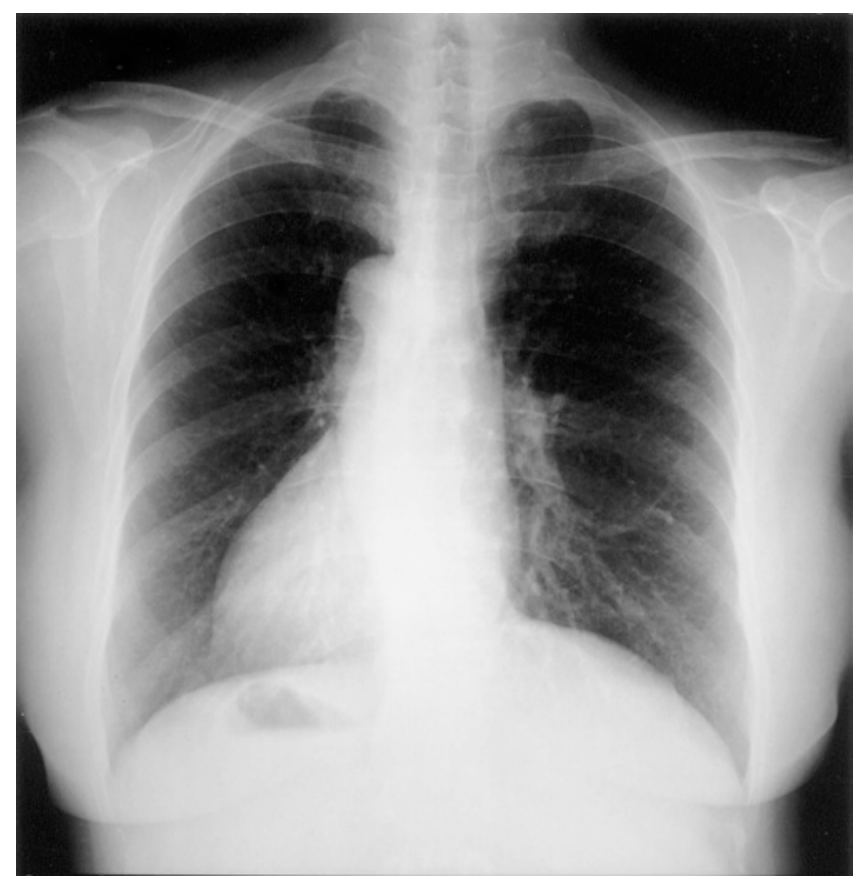

Figure 1. A chest radiograph showed a mirror image of the normal radiograph, with the cardiac shadow in the right hemithorax.

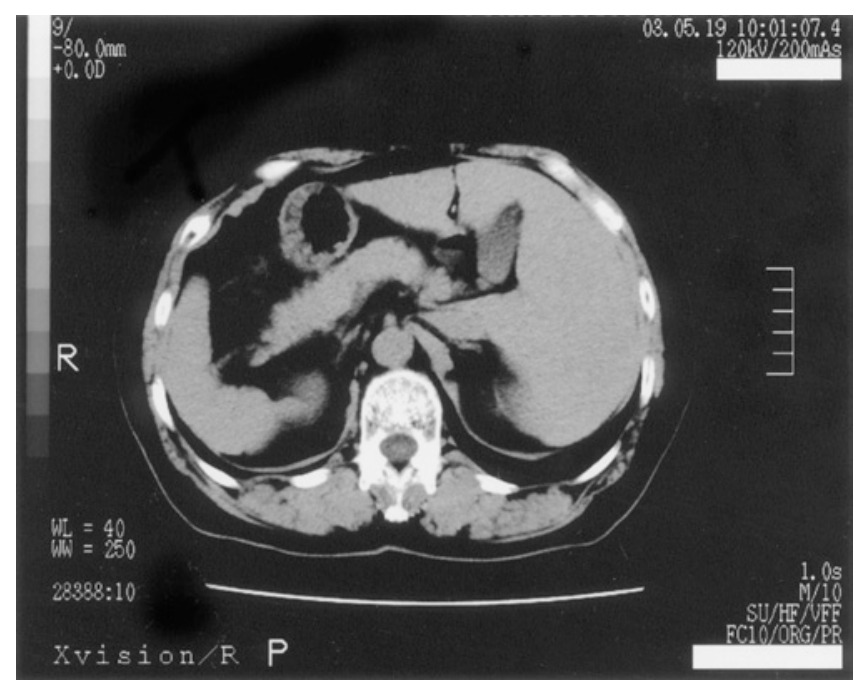

Figure 2. Abdominal CT demonstrated a mirror image of the normal CT, with the liver and gallbladder on the left side and the spleen on the right side.

A 52-year-old woman was admitted to our department for a close examination of liver dysfunction associated with type $\mathrm{B}$ hepatitis. A chest radiograph showed a mirror image of the normal radiograph, with the heart located at the right hemithorax (Fig. 1). A computed tomography (CT) of the abdomen also revealed the inversion of the normal CT, with the liver and gallbladder on the left side and the spleen on the right side (Fig. 2). She underwent a diagnostic laparoscopy which demonstrated situs inversus (Fig. 3). The hepatic surface pattern and histological findings of liver biopsy specimen were compatible with acute exacerbation of chronic hepatitis. Situs inversus totalis is a rare anomaly (incidence $1: 5,000$ to 1 : $10,000)$ characterized by the complete inversion of all abdominal and thoracic organs. In most cases, it is found incidentally by physicians during diagnostic imaging for unrelated conditions. 


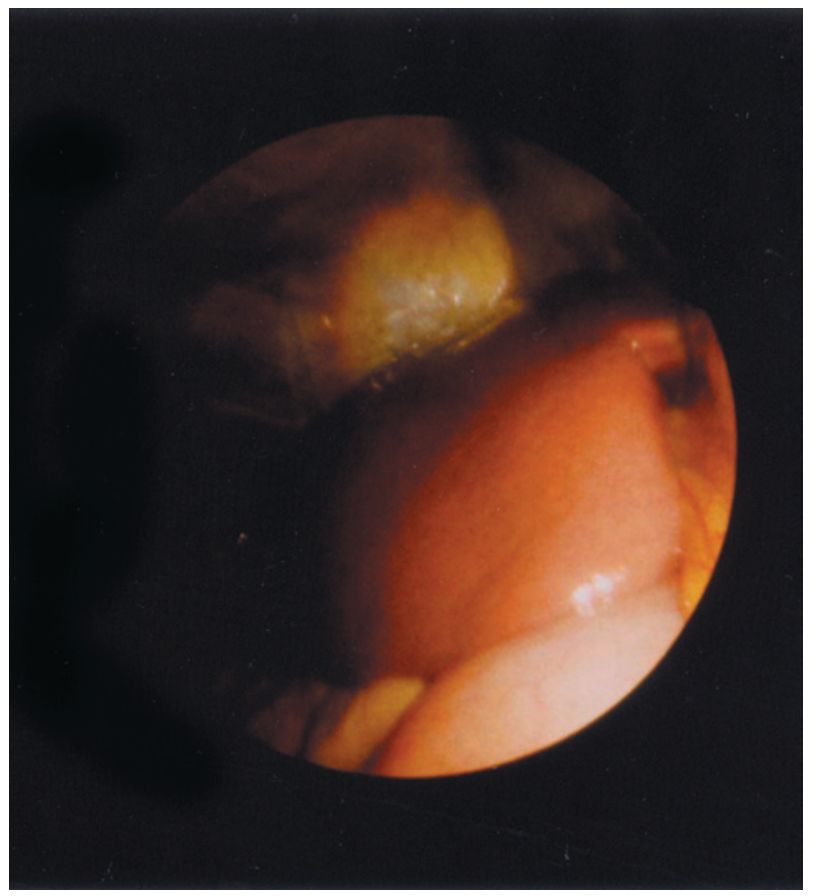

A



B

Figure 3. (A) Laparoscopic view of the right upper abdominal cavity beyond the round ligament demonstrated the inversion of the normal left hepatic lobe. The spleen was not visible. (B) Laparoscopic findings of the left upper abdominal cavity showed a mirror image of the right hepatic lobe and gallbladder.

Nobuyasu AIBA, Terumi TAKAHARA* and Takashi TsUKISHIRO**

From Seiyu Hospital, *the Third Department of Internal Medicine, Toyama Medical and Pharmaceutical University, Toyama and ** Department of Internal Medicine, Itoigawa General Hospital, Itoigawa

Received for publication October 18, 2004; Accepted for publication December 25, 2004

Reprint requests should be addressed to Dr. Nobuyasu Aiba, Seiyu Hospital, 103 Kamisenbyo-machi, Toyama 939-8134 